\title{
Corela
}

Cognition, représentation, langage

HS-34 | 2021

Les noms sous-spécifiés en français: du lexique au discours

\section{La place du nom fait parmi les noms sous-spécifiés}

\section{Danièle Van de Velde}

\section{(2) OpenEdition}

Journals

Édition électronique

URL : https://journals.openedition.org/corela/13899

DOI : 10.4000/corela.13899

ISSN : 1638-573X

Éditeur

Cercle linguistique du Centre et de l'Ouest - CerLICO

Référence électronique

Danièle Van de Velde, "La place du nom fait parmi les noms sous-spécifiés », Corela [En ligne], HS-34 I 2021, mis en ligne le 10 décembre 2021, consulté le 13 décembre 2021. URL : http://

journals.openedition.org/corela/13899; DOI : https://doi.org/10.4000/corela.13899

Ce document a été généré automatiquement le 13 décembre 2021.

\section{(c) (i) (3) (2)}

Corela - cognition, représentation, langage est mis à disposition selon les termes de la licence Creative Commons Attribution - Pas d'Utilisation Commerciale - Partage dans les Mêmes Conditions 4.0 International. 


\title{
La place du nom fait parmi les noms sous-spécifiés
}

\author{
Danièle Van de Velde
}

1 Dans ce qui suit, j'essaierai de décrire les propriétés sémantiques et pragmatiques du nom fait sur la base d'une analyse des structures syntaxiques dans lesquelles il entre au titre de nom sous-spécifié (NSS).

2 Un bref état de la question montrera que les propriétés le plus généralement reconnues aux NSS dans leur ensemble (contexte spécificationnel, absence de hiérarchie-être), permettent bien de les caractériser dans leur ensemble, mais non pas de les distinguer entre eux, si bien que les classements sémantiques qu'on en fait ensuite ne reposent que sur l'intuition.

3 Je proposerai donc de pousser l'examen syntaxique des structures dans lesquelles ces noms apparaissent au-delà de la simple distribution, ce qui permettra d'établir qu'elles sont en réalité de trois types, auxquels correspondent des propriétés sémantiques différentes des "shell nouns » correspondants. Le nom fait ainsi que les noms que j'appellerai « de faits qualifiés » entre dans l'un de ces trois types de structure, mais on peut montrer que même dans sa propre sous-classe, il occupe une position unique.

4 Une dernière partie exposera quelques-unes des propriétés remarquables qui font de fait un nom unique en son genre.

\section{Bref état de la question}

\subsection{NSS et constructions spécificationnelles}

5 Les noms sous-spécifiés sont nés, si on peut dire, comme «containers » dans le vocabulaire de Vendler qui distingue les «containers» nominaux, verbaux et adjectivaux, expressions contenant un "gap» susceptible d'être l'hôte d'une nominalisation (les que $P$ et les infinitives étant pour lui des nominalisations) : ainsi en va-t-il pour le nom fact dans les constructions du type :

$\mathbf{N}$ is $\mathbf{N}$ That he died is a fact 

Schmid, à qui elle est due, propose un schéma structural en trois parties :

shell noun (link) shell content

The thing is that I was told not to fight

de la phrase qu'ils "encapsulent/renferment», laquelle désigne "un fait, un événement ou un état de choses ». Ces noms sont "généraux» ou sous-spécifiés (unspecific), et abstraits (Adler 2017).

La sous-spécification elle-même consiste en une incomplétude sémantique qui en ellemême ne suffirait pas à caractériser la classe, si elle n'était en relation avec un type de construction dit spécificationnelle, dans laquelle une phrase ou un équivalent de phrase, complétant le nom, remplit en quelque sorte son sens. Ces constructions correspondent aux schémas de Vendler et de Schmid, avec quelques variantes.

Les propriétés distributionnelles des NSS se recouvrent donc (presque) complètement, puisqu'ils peuvent (presque) tous, comme fait lui-même, entrer dans les structures spécificationnelles $N$ est que $P$ et $N$ que $P^{1}$, où que $P$ peut être remplacé par de Vinf ou une nominalisation ${ }^{2}$ :

1) Le fait est que $P$ /Le fait que $P$

2) L'idée est de Vinf /L'idée de Vinf

3) Le problème est $N_{\text {déverbal }} /$ le problème de $N_{\text {déverbal }}$

Pour Vendler, qui a une conception très large de ce qu'il faut rassembler sous la notion de « container ", une nominalisation peut prendre la place de la que P comme c'est le cas dans le couple :

4) Le fait qu'il soit mort / le fait de sa mort

11 Et l'ordre sujet-attribut peut être inversé comme dans :

5) Sa mort est un fait

12 Dans la lignée de Vendler, on s'entend en général pour admettre que c'est seulement lorsqu'il entre dans (l'une au moins de) ces structures syntaxiques qu'un nom peut être considéré comme " sous-spécifié », ou plutôt « en emploi sous-spécifié ».

13 Mais tous les auteurs ne semblent pas s'accorder sur l'extension des constructions nécessaires et suffisantes pour qu'on puisse parler de NSS : Le Gallois n'inclut pas les nominalisations, ni, si je ne me trompe les constructions de type (4) où la que $P$ n'est pas en position attributive mais dans une position qui semble être celle d'un complément.

Dans ce qui suit j'adopterai une conception relativement large des structures spécificationnelles, en y incluant celles qui contiennent, à la place de la que $P$, une infinitive ou une nominalisation.

\subsection{NSS et hiérarchie-être}

15 En règle générale, un nom commun nomme la classe à laquelle appartient le denotatum, et il entretient avec d'autres noms plus spécifiques dont il contient en lui la signification, une relation hiérarchique de genre à espèce ou, comme disent Kleiber et Tamba (1990), une relation hiérarchique dénommée « hiérarchie-être ». Or fait n'est pas le nom d'une classe ou d'un genre, qui subsumerait des espèces de faits. Il ne prend place dans aucune « hiérarchie-être », comme il est bien démontré par Adler (2017), et cela est, selon l'auteure, l'une des caractéristiques essentielle de tous les noms sous- 
spécifiés. Il n'y a pas de classe des faits, comme il n'y a pas de classe des problèmes, des obstacles, des scandales, etc. ${ }^{3}$

A la démonstration de Adler, j'ajouterai un argument tiré du type de "relation-être " que le NSS entretient avec le nom qu'il encapsule.

Cette relation est exprimée par être dans les structures spécificationnelles attributives, et les NSS autorisent tous la substitution à être de constituer dans les structures où le NSS est en position d'attribut, et de consister dans celles où il est en position de sujet. Ainsi en va-t-il dans les cas suivants où le NSS est fait :

6) «L'existence de ces mails constitue (est) un fait nouveau »

7) «Le premier fait consiste dans (est /est constitué par) l'allongement de la durée

moyenne de la vie humaine »

8) Le fait nouveau consiste dans l'existence de ces mails

9) L'allongement de la durée moyenne de la vie humaine constitue le premier fait

Les mêmes substitutions sont possibles avec, semble-t-il, tous les autres NSS. En voici des exemples :

10) Sa paresse est / constitue un obstacle à sa réussite

11) L'idée de mon père est / (consiste en ce) que les terres soient vendues

12) Le scandale ${ }^{4}$ est / (consiste dans le fait) de donner à quelqu'un une occasion de faute

Or, dans les structures à attribut nominal, être indique généralement l'appartenance d'un individu à une classe lexicale préconstituée, ou d'une classe à une autre classe supérieure, comme quand on dit :

13) Un temple est un édifice religieux

Mais cette règle cesse de valoir dans les cas où la copule être peut être remplacée par le verbe constituer. Car ce verbe indique justement que le référent du sujet n'appartient pas à la classe dénotée par l'attribut, ce qui explique pourquoi on ne dira pas :

14) *Un temple constitue un édifice religieux

Un temple est en effet, selon sa définition même, ce qui revient à dire selon sa nature, un objet qui appartient à la classe des édifices religieux, qui est elle-même une sousclasse des édifices.

\section{Inversement le fait que l'on puisse dire :}

15) L'église constitue un asile inviolable

indique justement que l'église selon sa définition n'est pas un asile mais peut servir de, faire fonction de, valoir comme, être considéré comme... Bref, elle n'a pas la " nature » d'un asile parce que rien n'a en soi la nature d'un asile, ou que, en d'autres termes, il n'y a pas de classe lexicale des asiles.

24 Le NSS attribué à une subordonnée en que dans les structures spécificationnelles n'a donc pas de valeur classifiante pour le « shell content ", et cela semble propre à tous les NSS.

Mais s'il ne le classe pas, que fait-il ? Car, s'il est clair que le « shell content » donne un contenu au "shell noun", s'il est clair aussi que ce contenu, ayant la forme d'une proposition, ne peut être qu'un fait, un événement, ou un état de choses, le rôle de ce dernier (à part son rôle dans le discours, rôle fondamental mais que nous n'abordons pas ici) n'apparait pas clairement à première vue. Tout ce qu'on peut dire, c'est que le NSS « prend » un événement ou un état de choses et le présente d'une certaine façon, sous un certain angle, d'un certain point de vue. 


\subsection{Sous-classes sémantiques}

Sur la base des critères ci-dessus, on peut rassembler un grand nombre de noms (voir en particulier les tables 5, 6, 7, 8 de Schmid 2000). Mais leur sous-classification est ensuite exclusivement et immédiatement sémantique, effectuée au moyen de catégories entièrement intuitives, non fondées en syntaxe, qui sont les suivantes :

- Factual (fact, thing, point, problem, reason, difference, upshot)

- Linguistic (news, message, rumour, report, order, proposal, question)

- Mental (idea, notion, belief, assumption, aim, plan, decision)

- Modal (possibility, truth, permission, obligation, need, ability)

- Eventive (act, move, measure, reaction, attempt, tradition, trick)

Circumstantial (situation, contest, place, area, way, approach)

Je ne critiquerai pas en détail ce classement mais je relèverai seulement quelques points : le premier est qu'on y glisse assez souvent de la caractérisation du «shell noun » à celui du "shell content », ou inversement. Par exemple, les "shell nouns » dits « factual » rassemblent des noms dont le « shell content» est factuel, alors que leur signification propre ne l'est pas: ainsi en va-t-il pour reason et upshot dont le sens propre est causal, et qui reçoit un contenu factuel, puisque seul un fait peut être une cause.

Inversement, sous la rubrique "linguistic shell nouns" on trouve des noms qui dénotent bien en eux-mêmes et par eux-mêmes des entités linguistiques, alors même que leur «shell content » est factuel comme le montre, en français, pour un nom du même type un exemple tel que:

16) On vient d'apprendre la nouvelle que le président ne se représentera pas

Enfin, sous la rubrique "eventive», on ne trouve que des noms d'action - et pas de noms d'événements, pour la bonne raison qu'il n'existe pas de noms d'événements ${ }^{5}$.

Nous verrons que les parallélismes distributionnels, donc apparents, sont en partie trompeurs, en ce qu'ils permettent de regrouper des noms très différents par leurs propriétés sémantiques et syntaxiques. Pour regrouper ces noms en sous-classes, au lieu de passer directement de la distribution au sens, nous approfondirons le type de relation syntaxique que les NSS entretiennent avec les phrases qui peuvent leur être liées par la copule, ou leur être adjointes comme compléments.

\section{Typologie des NSS}

\subsection{La relation entre les NSS et leur complément}

Cependant, le fait que tous les NSS partagent la propriété de n'être pas des noms de classe lexicale, et ont par ailleurs la même distribution, en ce que tous ils entrent dans les différents types de constructions dites spécificationnelles, ne suffit pas à en faire une classe homogène. La distribution masque en effet un fait syntaxique, ce qui veut dire aussi sémantique, important : il s'agit de la relation entre la que $P$ (ou son substitut) et le NSS.

Soient les phrases suivantes :

17) Le fait que tu sois parti lui a paru bizarre

18) La crainte que tu partes lui est venue hier 
La substitution à la subordonnée en que d'une infinitive sous condition d'identité ${ }^{6}$, ou d'une nominalisation, si un nom dérivé du verbe de la complétive est disponible, entraîne l'insertion de la préposition de, ce qui semble correspondre à la formation d'un génitif, et dans ce cas d'un génitif complément du nom :

19) Le fait d'être parti lui a paru bizarre

20) Le fait de ta venue lui a paru bizarre

On observe la même chose avec le $\mathrm{N}$ crainte :

21) La crainte de partir lui est venue hier

22) La crainte de ton départ lui est venue hier

Ce sont des parallélismes de ce type qui conduisent les auteurs à rapprocher et même à assimiler les structures associées ici à crainte, et celles associées à fait, en tant que " nominal constructions that show dependency phenomena similar to those found with verbs » (Schmid 2007, 315).

Le même auteur propose comme exemples de ces noms l'échantillon suivant :

a) $\mathrm{N}+$ that clause : the fact that abstract nouns are difficult to pin down...

b) $\mathrm{N}+$ to-infinitive : the idea to illustrate the patterns investigated...

c) $\mathrm{N}+\mathrm{BE}+$ that-clause : the problem is that there is a lot to study.

d) N+BE+to-infinitive : the solution is to focus on a bunch of examples.

Pour des raisons de place je n'examinerai que les constructions de type (a) et (b), qui d'ailleurs paraissent interchangeables, puisque fact, comme fait, peut être suivi d'une infinitive sous condition d'identité et que, inversement idea, comme idée, peut être suivi d'une complétive :

23) The fact to have studied matters a lot for young people /Le fait d'avoir étudié est important pour les jeunes gens

24) The idea that social conditions are fundamental causes of diseases is gaining credibility / L'idée que les conditions sociales sont une des principales causes de maladie gagne du terrain

Nous ajouterons à ces deux noms, entrant dans les mêmes constructions, le nom possibilité, qui appartient encore à un autre type et entretient avec son " complément " encore une autre relation. Ce nom est dérivé d'un adjectif qui appartient chez Vendler à la catégorie des containers adjectivaux avec «likely, probable, possible, certain, and opposites ». On trouve également chez Schmid une catégorie de containers " modaux ».

Il peut en effet être tentant, sur la base de certaines définitions des modalités, de rapprocher la factualité de la possibilité et de la nécessité, selon que l'on considère ou non que l'assertivité est un mode, ce qui est souvent contesté sur la base du fait que c'est une propriété non marquée. J'essaierai de montrer comment, dans la langue, ce rapprochement, quoique en partie justifié, ne permet pas l'assimilation totale de l'assertivité à une modalité comme les autres. Voici, pour compléter notre échantillon, des constructions spécificationnelles avec possibilité :

25) La possibilité que tu partes lui fait peur

26) La possibilité de tomber lui fait peur

27) La possibilité d'une chute lui fait peur

4 Le parallélisme distributionnel entre les trois types de noms semble donc à première vue complet, mais la syntaxe, en l'occurrence la relation syntactico-sémantique entre la phrase en que ou son substitut infinitival ou nominal et le nom que cette phrase " complète » n'est pas du tout la même selon que ce nom appartient au type (1) fait, (2) crainte, ou (3) possibilité. En fait, c'est seulement dans le cas de crainte (ou idée, désir, 
appréhension, regret, et autres), qu'on peut parler, comme le fait Schmid, de " phénomènes de dépendance semblables à ceux qu'on trouve avec les verbes ».

En atteste en particulier la pronominalisation de ce qui apparaît dans les trois cas comme un complément, mais qui n'en est véritablement un qu'avec crainte, et qui mérite encore ce nom avec possibilité, mais dans une acception différente, mais pas avec fait. Les compléments des noms de la classe (2) se pronominalisent sans difficulté par en:

28) La crainte / l'idée lui en est venue hier

Mais avec fait, la pronominalisation par en est tout à fait impossible et on ne dira pas :

29) *Le fait lui en a paru bizarre

Les noms du type (3) autorisent également la pronominalisation par en, mais ce en n'en est pas moins différent de celui qu'on trouve en (28). Dans la phrase :

30) «La possibilité (du transfert de fonds) en est donnée par la loi »

le pronom en équivaut lui aussi à un génitif, mais c'est le génitif qui marque la relation entre une entité et une de ses propriétés : la possibilité d'un transfert de fonds, qui est un événement, est une propriété de celui-ci, comme la beauté d'un tableau est l'une de ses propriétés.

Comment interpréter ces faits? Ils nous conduisent à la conclusion qu'il existe (au moins) trois types de relations entre une NSS et son complément. Lorsque ce dernier est pronominalisable par en, il indique soit une relation de possession (30) soit une relation de complémentation véritable, au sens à la fois intuitif et traditionnel où le complément complète le $\mathrm{N}$ (ou V ou A) dont il est complément (28).

Commençons par la relation de complémentation : le cas de la crainte. Si on éprouve de la crainte, celle-ci a nécessairement un objet, elle est crainte de quelque chose. Le complément dans les phrases (18) et (21-22) indique l'objet de la crainte et, quoique le nom crainte soit devenu par la nominalisation un nom véritable, il retient quelque chose de sa nature verbale, et son complément entretient avec lui le même rapport que le verbe craindre avec ses arguments.

Il en va de même avec tous les noms dérivés de verbes (ou même exprimant une idée verbale comme idée) et exprimant un acte mental (idée, pensée) ou ce qu'on peut appeler une «attitude propositionnelle»: certitude, impression, désir, ou encore un "sentiment » : crainte, espoir, sentiment... Ces noms ont pour dénotation le contenu de l'acte mental ou de l'attitude ou du sentiment que le verbe apparenté signifie. Dans une phrase telle que :

31) Je projette de partir

49 (où le de est explétif, cf. je le projette), le verbe à l'infinitif dénote l'objet intentionnel de l'acte mental de projeter. C'est le cas encore dans l'expression nominale :

32) Mon projet de partir

Le rôle du nom est alors de relier le contenu de la que $P$ à un participant du discours, dont il constitue un objet intentionnel.

51 Dans le cas de la possibilité, le NSS attribue au contenu de son complément qui a la signification d'une situation (état de choses ou événement), une propriété. Cette propriété établit elle aussi un lien, mais qui rattache le contenu de la que $P$, considéré en lui-même, au réel. La possibilité de sa chute, par exemple, caractérise le rapport que l'événement sa chute entretient avec le réel, puisque en lui-même il n'est ni réel ni 
irréel ${ }^{7}$, rapport tel que sa réalité n'est pas exclue. Le rapport institué par inévitable serait que la non-réalité de l'événement est exclue, et ainsi de suite. servent à attribuer à la dénotation de la proposition ou de la nominalisation non pas exactement une propriété, mais une autre (nouvelle) nature, en les recatégorisant comme " fait » "scandale », etc., et que la préposition de est l'instrument copulatif qui permet d'établir cette relation.

\subsection{Fait parmi les autres NSS}

59 Je propose donc de distinguer parmi les NSS généralement reconnus comme tels, d'une part les noms que j'appelle de faits ${ }^{8}$ et de "faits qualifiées" (fait, problème, scandale, obstacle), d'autre part ceux qu'on pourrait appeler «noms d'objets intentionnels » tels que désir, crainte, projet etc. lesquels, tout en constituant des nominalisations achevées, 
conservent la valence correspondant à la structure argumentale du verbe dont ils dérivent ${ }^{9}$, enfin les noms modaux, proches des premiers mais qui s'en distinguent cependant.

\subsubsection{Le rapport des faits aux événements} comportent un temps. Or un événement à lui seul ne peut pas être dénoté par une phrase car il est dépourvu d'ancrage temporel ; c'est seulement lorsque l'événement se réalise qu'il reçoit un ancrage temporel. Les seules expressions linguistiques qui en elles-mêmes peuvent dénoter des événements sont des nominalisations et encore, à condition qu'elles ne contiennent pas de temps. C'est pourquoi le sujet de la première des deux phrases suivantes dénote un fait, mais celui de la seconde un événement :

43) L'effondrement de l'Allemagne à ce moment-là avait été prévu

44) L'effondrement de l'Allemagne eut lieu à ce moment-là constituant circonstanciel est possible, comme dans :

45) L'événement de (que fut) l'effondrement de l'Allemagne eut lieu à ce moment-là

\subsubsection{P, Que $P$, Le fait que $p$}


locuteur n'est pas intervenue. C'est pourquoi le sujet de la phrase suivante ne dénote pas un fait, à la différence du sujet de la seconde :

46) Qu'il ait réussi son examen m'étonnerait beaucoup

47) Qu'il ait réussi son examen ne m'étonne pas

Considérons la batterie de phrases suivantes :
48) Le fait est qu'il a réussi
49) Le fait important est qu'il a / qu'il ait réussi
50) Le fait important est sa réussite ${ }^{11}$

71 Le verbe être, dont il a été dit qu'il pouvait être remplacé par consister lorsque la complétive est en position d'attribut, vérifie cette propriété en (52-53), mais pas en (51) :

51) *Le fait consiste en ce qu'il a réussi ${ }^{12}$

52) Le fait important consiste en ce qu'il a réussi

53) Le fait important consiste dans sa réussite

Autre indice de figement: la liaison (phonétique) entre fait et est est absolument obligatoire quand il n'y a pas de modifieur, et elle s'accompagne d'une pause après le verbe. Elle ne l'est pas, au contraire, quand un modifieur est présent, et s'il y a une pause, elle sera après fait :

54) «Le principal fait est qu'on a trouvé une autre possibilité de trouver une planète habitable » 
73 Ajoutons enfin qu'après le nom modifié on a le choix, dans la complétive, entre le subjonctif et l'indicatif, ce qui n'est pas le cas après le nom non modifié, ce qui nous permet de conclure qu'il existe une expression le fait est, non modifiable, qui est une sorte de renforcement de la force assertive de la phrase ${ }^{13}$.

\subsection{Est un fait, C'est un fait} factualité affirmée dans le prédicat n'est pas déjà incluse dans la signification de leur sujet : ce sont des jugements synthétiques. On pourrait en effet très bien attribuer au sujet de ces phrases un prédicat qui caractérise leur référent comme virtuel. Ainsi dans :

58) Qu'il ait réussi / Sa réussite est impossible

55) Le fait qu'il ait réussi / de sa réussite l'a beaucoup réconforté

\section{l'article défini qui précède le nom fait est justifié par la structure qui le suit, et qui a une} valeur spécificationnelle: on peut le faire apparaître facilement par les gloses contenues en (56):

56) Le fait qui consiste en ce qu'il a réussi / dans sa réussite l'a beaucoup réconforté

Le présupposé commun aux deux groupes nominaux sujets de ces phrases est donc:

57) Qu'il ait réussi / Sa réussite est un fait

Au contraire, en (59) ci-dessous, la seconde phrase ne fait qu'attribuer à la dénotation de la première une propriété (être un fait) qu'elle possède déjà en tant qu'assertion.

59) Il a réussi son examen. C'est un fait

79 On vérifie ainsi que l'enchâssement ou la nominalisation ôte ou plus exactement suspend l'ancrage dans le réel que les phrases ont lorsqu'elles sont énoncées sous la forme d'indépendantes assertives.

80 Il existe cependant des contraintes sur la phrase (ou l'équivalent de phrase) sujet de être un fait, et qui expliquent en particulier un contraste comme celui qui oppose les phrases suivantes :

60) Qu'il réussisse est impossible

61) *Qu'il réussisse est un fait ainsi que celui entre (61) et (62) :

62) Sa réussite est un fait

L'explication peut se résumer à ceci que dans une phrase en que sujet de être un fait, le temps ne peut pas être suspendu comme il peut l'être dans d'autres contextes. Or en (61) le subjonctif est à la forme dite « subjonctif présent ", mais en fait il ne marque pas d'ancrage temporel dans le présent. C'est donc l'absence de temps qui rend la phrase inacceptable, d'où la conclusion qu'une phrase en que doit, pour pouvoir être dite être un fait, avoir un ancrage temporel.

83 Cependant cette condition, absolument nécessaire, n'est pas encore suffisante, puisque certaines que $P$, d'interprétation virtuelle, sont marquées pour le temps comme c'est le cas de la phrase suivante :

63) Qu'il soit arrivé à l'heure serait très étonnant 
où le subjonctif est combiné avec une marque aspect
localisation du procès dans le passé, comme dans :

64) Qu'il soit arrivé à l'heure nous a tous étonnés

Nous arrivons donc à la conclusion que la phrase enchâssée, même si son contenu met en relation l'événement ou l'état de choses qu'elle signifie avec un temps déterminé, n'a pas encore forcément d'interprétation factuelle: il y faut un constituant supplémentaire, que certains syntacticiens appellent "force», qui correspond au " mode » de la tradition grammaticale, et dont on dit que c'est "le mode des phrases énonciatives... le mode du fait», par opposition au subjonctif qui «indique que le locuteur ne s'engage pas sur la réalité du fait» (Grevisse) ${ }^{14}$. Ce que j'appelle ici un constituant, parce que je pense qu'il a une existence en syntaxe, dans la partie la plus " haute » de la périphérie gauche de la phrase, est justement ce qui est parfois lexicalisé par le nom fait et qui peut prendre place à gauche de la conjonction, comme en (65) :

65) Le fait qu'il soit arrivé à l'heure nous a tous étonnés

La «force » doit être comprise ici comme «force illocutoire » - et on aura remarqué que dans le texte de Grevisse "énonciation » et « fait " sont liés, comme deux faces d'une même chose, ce qui suggère fortement qu'un fait n'est un fait que s'il est assumé comme tel par (au moins) un sujet parlant. Il existe d'autres indices de ce caractère phénoménologique de la notion de fait par exemple l'équivalence dans la pratique ordinaire entre fait accompli, où l'épithète ne concerne que le fait, et d'autres expressions comme fait acquis (« reconnu sans contestation» Robert), fait établi et autres, qui rapportent la factualité à un sujet. Au nombre de ces indices on comptera aussi l'étrange « fait nouveau » qui signifie la plupart de temps non pas «fait basé sur un événement qui vient de se produire", mais "fait nouvellement parvenu à la connaissance » de l'énonciateur et d'autres.

Le rôle du constituant "force " quand celui-ci est assertif, correspond donc, toutes choses égales par ailleurs, à celui de l'article défini devant un nom, puisque l'un et l'autre comporte un présupposé d'existence du référent: la phrase assertive présuppose la réalité de l'événement ou de la situation assertée, comme l'article défini présuppose la réalité le l'objet visé par le groupe nominal.

Quant à cette réalité, elle est acquise, ce qui veut dire qu'un événement ou une réalité peuvent « devenir » des faits.

\subsection{Devenir un fait, être désormais un fait}

89 Ces deux expressions sont généralement prédiquées d'une éventualité : la première la saisit dans le moment de son actualisation, comme on le voit dans les exemples suivants :

66) «L'hypothèse d'un peuplement des Amériques bien antérieur à ce qu'on dit devint un fait » (Google)

67) «C'est là que furent déplacées les industries lorsque l'invasion devint un fait » (Google)

90 Ce que disent les phrases de ce type c'est qu'il y a un moment du temps où une hypothèse, une crainte, un risque, devient un fait, et que par conséquent il y a un moment de l'émergence du fait comme tel. A partir de ce moment, le fait est établi, acquis, si bien qu'on peut dire que « être un fait » est l'état résultant d'un changement, 
celui que dénote l'expression "devenir un fait», passage illustré dans l'exemple suivant :

68) (Que François Hollande se représente et trahisse son parti) «ce n'est plus un risque, c'est (désormais) un fait » (Google)

91 On ne peut donc pas nier qu'il y ait un rapport étroit entre les faits et le temps, puisque les faits apparaissent dans le monde à des moments déterminés, où une situation ou un événement que l'on s'imaginait comme possible, que l'on craignait, que l'on souhaitait, devient une réalité, se réalise.

Mais il est aussi non moins indéniable que les faits, une fois devenus ce qu'ils sont, échappent au temps comme Vendler, dans une discussion célèbre avec Austin, le soutient. Ils n'apparaissent, en somme, que pour disparaître.

\section{Conclusion : Vendler contre Austin}

93 Le débat est en réalité à trois : d'un côté Strawson opposé à Austin, Vendler prenant dans la discussion qui les oppose le parti de Strawson. La question initiale que ces philosophes se posent est: les faits sont-ils "genuinely-in-the-world» ou non? La réponse de Vendler comme celle de Strawson est négative : l'un et l'autre admettent que les événements sont « dans le monde », mais refusent d'y admettre les faits.

Le principal argument linguistique de Vendler (1967) lorsqu'il introduit pour la première fois la notion de "container context " est qu'on dit bien The collapse of the Germans was an event mais pas *was a fact ${ }^{15}$. Vendler ne dit pas pourquoi il en est ainsi, mais la réponse à cette question est peut-être aussi simple que celle-ci : si, pour un événement, "être un fait ", ou si on veut "devenir un fait", veut dire changer de nature dès l'instant où il est "survenu", il est assez clair que ce qui est une fois survenu ne peut plus, étant donnée l'irréversibilité du temps, ne pas être survenu, ou si on veut, est survenu pour toujours. On peut répéter ici, avec Sartre, que les faits sont « irrémédiables ». Il y a dans cette conclusion, du côté du monde, l'équivalent de ce que l'on peut dire, du côté du langage, des énoncés objectifs ${ }^{16}$ qui ont été une fois vrais : ils le seront à jamais. Leur vérité ne peut pas être défaite, comme ne le peut pas non plus l' " être survenu » des événements, ou l'avoir été des états de choses. Pour cette raison, on peut dire que "être un fait » est un prédicat de type "individual level », qui dit quelque chose sur la nature, ou sur l'essence d'un événement ou d'un état de choses accompli. Du coup, aussi paradoxal que cela puisse paraître, tout événement ou état de choses, à peine entré dans le réel, donc entré dans le temps (et l'espace), échappe au temps (et à l'espace).

Cette particularité n'est pas partagée, cependant, par les noms dénotant des faits "qualifiés» tels que problème, obstacle, scandale qui peuvent très bien s'employer au passé (cela fut un problème /un scandale / un obstacle...). C'est que ce qui est alors dans la portée du temps n'est pas le caractère factuel mais le caractère problématique, ou scandaleux, ou d'empêchement, du référent - exactement comme lorsqu'on dit d'une personne vivante qu'elle a été "une belle femme ", on ne veut pas dire qu'elle n'est plus une femme, mais qu'elle n'est plus belle.

Unique, donc, même à l'intérieur de sa propre sous-classe, le nom fait l'est encore en ceci qu'il est, d'une certaine façon, tout à fait inutile (sauf, bien entendu, comme outil énonciatif - et en tant que tel il est irremplaçable) : en effet, il n'est besoin, pour 
« dire » qu'une situation ou un événement constitue un fait, que de former une phrase assertive complète. Le nom fait explicite, et fait porter l'emphase sur, une propriété de la dénotation des phrases qui est tellement fondamentale qu'elle est déjà fondée dans leur structure syntaxique. C'est ce que fait déjà de manière exemplaire l'expression le fait est que placée devant une phrase assertive, qui n'a pas besoin d'elle pour être comprise comme énonçant un fait.

\section{BIBLIOGRAPHIE}

Adler, S. (2017). « Les noms généraux -« Shell Nouns »- participent-ils à une lecture taxinomique de type hiérarchie-être ? ». Syntaxe et Sémantique 18, 45-66.

Adler, S. (2014). « Évaluation, référence et noms généraux attitudinaux ». Langue Française 184, 93-108.

Austin, J., L. (1961). « Unfait to Facts ». In Philosophical Papers. Oxford : Clarendon Press.

Gardies, J.-L. (1983). « Tentative d'une définition de la modalité ». In J. David \& G. Kleiber (éds), La notion sémantico-logique de modalité. Paris : Klincksieck, 13-24.

Godard, D. \& Jayez, J. (1996). « Quels sont les faits ?». In M. Plénat, M. Aurnague, A. Condamines, J.P. Maurel \& C. Molinier (éds), L'emprise du sens : structures linguistiques et interprétations. Mélanges de syntaxe et de sémantique offerts à Andrée Borillo. Amsterdam : Rodopi, 117-136.

Heyvaert, L. (2003). A Cognitive Approach to Nominalization in English. Berlin, New York: Mouton De Gruyter.

Huyghe, R. (2018). «Généralité sémantique et portage propositionnel : le cas de fait ». Langue Française 198, 35-50.

Kleiber, G. (1984). « Dénomination et relations dénominatives ». Langages 76, 77-94.

Kleiber, G. \& Tamba, I. (1990). « L’hyponymie revisitée : inclusion et hiérarchie ». Langages 98, 7-32.

Legallois, D. (2006). « Quand le texte signale sa structure : la fonction textuelle des noms sousspécifiés ». Corela HS-5 ( http://journals.openedition.org/corela/1465)

Legallois, D. (2008). « Sur quelques caractéristiques des noms sous-spécifiés ». Scolia 23, 109-127.

Milner, J.-C. (1978). « Particularités diverses des expressions qualitatives. Appendice ». In De la syntaxe à l'interprétation. Paris : Le Seuil.

Schmid, H.-J. (2007). « Non-Compositionality and Emergent Meaning of Lexico-Grammatical Chunks : a Corpus Study of Noun Phrases with Sentential Complements as Constructions ». ZAA 55.3, 313-340.

Schmid, H.-J. (2000). English Abstract Nouns as Conceptual Shells : from Corpus to Cognition. Berlin : De Gruyter. 
Van de Velde, D. (2001). « Les structures nominales dénominatives ». In D. Amiot, W. De Mulder \& N. Flaux (éds), Le syntagme nominal, syntaxe et sémantique. Arras : Artois Presses Université,

289-311.

Van de Velde, D. (2006). « Le fait de et l'action de : quelle différence? ». In M. Tenchea et A.Tihu (éds), Prépositions et conjonctions de subordination, syntaxe et sémantique. Timisoara : Exelsior Art, 263-275.

Van de Velde, D. (2013). « La construction le fait que P constitue-t-elle une structure binominale dénominative? ». Cahiers de Lexicologie 103, 145-163.

Van de Velde, D. (2018). Faits et Causes. Villeneuve d'Ascq : Presses Universitaires du Septentrion. Vendler, Z. (1967). « Facts and Events ». In Linguistics in Philosophy. Ithaca, New York : Cornell University Press.

Vendler, Z. (1968). Adjectives and Nominalizations. The Hague: Mouton.

\section{NOTES}

1. Pour Schmid (2000) il faut et il suffit que le nom puisse entrer dans au moins l'une de ces deux structures.

2. Un relecteur anonyme fait remarquer qu'un certain nombre de NSS, dont fait, n'ont pas d'emploi générique, ce qui est en effet intrigant. Je ne vois pas d'autre explication à ce fait que celle de Vendler, (1967, «Singular Terms ») qui, pour maintenir l'idée que l'emploi de l'article défini suppose toujours un dispositif restrictif, propose de « dériver » the tiger de the (animal that is a) tiger. Cette solution implique bien sûr l'existence d'un nom superordonné. D'où selon Vendler la difficulté à employer des noms très généraux, comme object, avec l'article défini générique.

3. On peut cependant remarquer que certains noms généralement considérés comme NSS : désir, crainte, par exemple, s'ils sont eux-mêmes des noms d'espèces dernières, appartiennent à des genres - celui, en l'occurrence, des affects, qui appartiennent eux-mêmes à celui des états psychologiques. Ils appartiennent donc à une hiérarchie-être, mais ils en sont les termes ultimes.

4. Je considère obstacle et scandale comme des NSS quoique la seule construction spécificationnelle non copulative dans laquelle ils entrent couramment soit une nominalisation, mais qui est une réduction de phrase, puisqu'elle a une interprétation factuelle. On trouve cependant assez couramment des exemples de queP après scandale ( le scandale qu'il faille faire appel à la générosité du public », « le scandale que tu sois l'amant de ma sœur ») - et, surtout, il y a d'innombrables exemples de ces deux noms dans la construction le scandale que constitue le fait que $P$.

5. Les noms dits "d'événements" considérés en eux-mêmes sont des noms généralement déverbaux signifiant des procès, ponctuels ou non, des activités, des actions, transitives ou non, bref, toutes les variétés qu'on peut faire entrer sous le concept général d'action. Ces noms fournissent le noyau nominal d'expressions susceptibles de dénoter des actions, mais aussi des événements ou des faits, selon le contexte. Ainsi le nom intervention dénote-t-il une action, un événement et un fait dans les trois contextes suivants : il a fait hier une intervention remarquée / sa prochaine intervention aura lieu dans trois jours / on ne s'attendait pas à son intervention.

6. La condition de la réduction d'une quP à une infinitive est que le sujet (non exprimé) du verbe de la subordonnée à l'infinitif soit identique à un groupe nominal, souvent mais pas exclusivement le sujet, de la proposition principale. Il s'agit donc d'une condition syntaxique, et l'infinitif n'a pas d'interprétation sémantique spécifique, comme semble le penser Schmid. 
7. Je précise que, contre Godard et Jayez (1996), je maintiens une solide distinction entre événement et fait en dépit des glissements qui se produisent parfois de l'un à l'autre dans le langage courant, et en particulier celui qui fait dire que «les faits se sont produits »: ce glissement, le seul relativement fréquent, s'explique parfaitement bien sur la base de l'idée que les faits sont le fruit des événements. Mais on ne dira jamais d'un fait basé sur un état de choses qu'il a « duré » (remarque due à Vendler (1967, ch.5) et reprise par Godard et Jayez).

8. Il n'y a en réalité qu'un nom de fait : fait, les autres sont des noms de faits qualifiés : problème = fait problématique, scandale $=$ fait scandaleux, etc.

9. Une étude plus approfondie devrait distinguer deux emplois sensiblement différents de ces noms (ou d'une partie importante d'entre eux en tout cas) en fonction des structures dans lesquelles ils apparaissent. En (i) et (ii), par exemple, les propriétés aspectuelles du nom désir ne sont pas les mêmes, et on peut dire que c'est seulement en (ii) que ce nom est sous-spécifié : (i) Son désir de tout connaître dans le domaine est irrépressible ; (ii) Son désir est de tout connaître dans le domaine. C'est seulement en (ii), en effet que désir a le sens résultatif de " chose désirée ", puisque c'est seulement en (ii) et pas en (i) qu'on peut le traduire par « ce qu'il désire ». C'est donc seulement en (ii) et pas en (i) qu'on peut l'appeler nom « sous-spécifié ». Il reste néanmoins dans cet emploi un nom intensif, puisque l'interprétation résultative «ce qu'il désire » n'exclut pas une modification du prédicat verbal (« ce qu'il désire beaucoup / le plus... »

10. On peut objecter à cette affirmation, comme le fait un relecteur, des reprises anaphoriques telles que «Le ministre a remis sa démission. Cet événement... ». Mais on peut aussi bien avoir : «Le ministre a remis sa démission. Cet acte... ». Cela ne prouve pas que la phrase assertive dénote un acte, mais que l'anaphore peut «extraire » d'une phrase complète des constituants plus ou moins larges interprétables comme signifiant des actions ou états ou des événements ou situations.

11. On ne peut avoir ni après fait ni après fait important une infinitive, car le reste de la phrase ne contient aucun nom coréférentiel du sujet qui satisfasse à la condition d'identité à laquelle est soumise la réduction d'une phrase à l'infinitif. Ainsi, la possibilité de le fait de réussir l'a dopé (exemple d'un relecteur) repose sur le fait que la principale contient un pronom (le) qui peut être coréférentiel du sujet non exprimé de l'infinitif.

12. En réalité, cette phrase n'est inacceptable que si l'article défini ne peut pas s'interpréter comme anaphorique. Comparez: (i) Un fait nouveau vient ruiner tout ce qui a été dit de lui. Le fait consiste en ce qu'il a réussi / dans sa réussite, (ii) On dit qu'il n'est pas fait pour faire ces études. *Mais le fait consiste en ce qu'il a réussi.

13. Reste la question de la nature de l'article défini devant fait dans l'expression le fait est que: quel fait? Il n'y a pas de réponse à cette question parce que aucun fait, en réalité, n'est d'abord posé. Je proposerais de voir ici un usage explétif du défini, comme il y en a d'autres en français en rapprochant aussi l'expression de son équivalent italien le plus courant : fatto sta que, et d'une autre expression française synonyme : il est de fait que. L'usage de ces deux expressions comme particules de renforcement de l'assertion est confirmé par le très grand nombre d'occurrences dans lesquelles la phrase qu'elles introduisent est paradoxale confrontée à la précédente : Je suis belle, j'ai cet avantage pour lequel madame de Staël eût tout sacrifié; et pourtant il est de fait que je m'ennuie à mourir.

14. D'innombrables faits linguistiques obligent à distinguer au moins pour le français, un mode qu'on pourrait appeler "grammatical » d'un autre qu'on pourrait appeler «logique ", et qui s'appelle souvent dans la littérature «modalité ». C'est ce dernier qui correspond au constituant «force» de la syntaxe générative.

15. Cela vaut aussi en français, sauf évidemment quand le passé est requis pour des raisons formelles de concordance, dans les discours indirects libres en particulier.

16. Voir Van de Velde $(2015,79)$. Par « objectif» il faut entendre un énoncé qui ne comporte pas de repères spatio-temporels déictiques, énoncés dont la vérité dépend des conditions 
d'énonciation, mais seulement des noms propres de personnes, de temps et de lieu. Soient les deux énoncés: (i) Mon père a publié un livre aujourd'hui ; (ii) Napoléon a gagné en 1800 à Marengo sa première bataille importante. On peut dire que seul le second, s'il a été vrai une fois, le sera toujours, quelles que soient les conditions de son énonciation. C'est un énoncé objectif.

\section{RÉSUMÉS}

Le présent travail se concentre sur les propriétés sémantico-syntaxiques du nom fait à l'exclusion, par conséquent, de ses usages énonciatifs, qui devraient constituer un volet complémentaire de la présente étude. La première partie du texte refait un bref historique de la définition des NSS et ajoute un nouvel argument à ceux de Adler (2017) pour appuyer l'idée que ces noms ne participent d'aucune "hiérarchie-être ». La seconde propose une répartition des NSS en trois sous-classes sur la base de critères syntaxiques, qui font apparaître que toutes les structures spécificationnelles ne sont pas identiques, en dépit des apparences. Fait et les noms de «faits qualifiés » constituent une de ces sous-classes. Mais il est montré dans la troisième partie que fait occupe même dans sa propre sous-classe une position tout à fait unique.

This article presents a study of the semantic and syntactic properties of the noun fait (fact), disregarding, for sake of brevity, the pragmatic and enunciative peculiarities of its use in discourse. In the first part, I recall what is already known of the specificational constructions typical of the NSS, adding only a new argument in favor of the idea (Adler 2017) that these nouns do not enter any «Be-Hierarchy ». In a second part, I show that a simple distributional syntactic description of the constructions the NSS enter, cannot be a good basis for a semantic (non intuitive) classification. In fact, these constructions are of three types, each corresponding to a semantic class. Fait and the nouns of «qualified facts " constitute one of the three classes, but even inside its own class, fait has a unique and singular position.

shell nouns, facts, nominal lexicon, under-specification, specificational constructions

\section{INDEX}

Mots-clés : noms sous-spécifiés, faits, lexique nominal, under-spécification, constructions spécificationnelles

\section{AUTEUR}

\section{DANIÈLE VAN DE VELDE}

Université Lille 3, STL 